\title{
What Symptoms Are More Important for Korean Patients in Knee Osteoarthritis? Development and Validation of the Korean Knee Score
}

\author{
Jeong Ku Ha, $\mathrm{MD}^{1}$, Jin Goo Kim, MD, $\mathrm{PhD}^{1}$, Myung Chul Lee, $\mathrm{MD}, \mathrm{PhD}^{2}$ and Joon Ho Wang, MD, $\mathrm{PhD}^{3}$; \\ Research Committee for Development of a Novel Knee Evaluation System of Korean Knee Society \\ Department of Orthopedic Surgery, ${ }^{1}$ Seoul Paik Hospital, Inje University College of Medicine; ${ }^{2}$ Seoul National University College of Medicine; ${ }^{3}$ Samsung Medical \\ Center, Sungkyunkwan Unviersity School of Medicine, Seoul, Korea
}

\begin{abstract}
Purpose: The purpose of this study was to develop and validate a novel knee evaluation instrument, the Korean Knee Score (KKS), to reflect a floor life style with high knee flexion. In addition, we aimed to assess the importance of high knee flexion activity for Korean patients.

Materials and Methods: The KKS was developed following the guidelines of the International Society of Arthroscopy, Knee Surgery and Orthopaedic Sports Medicine. During development step, generated items were asked to 50 patients to determine importance and obtain an impact score for each item. A total of 634 patients were included in the validation survey.

Results: Forty one items were generated and impact scores of each item about floor life style were higher than other items. The KKS exhibited excellent reliability (intraclass correlation coefficient $=0.931$ ) and strong internal consistency (Cronbach's $\alpha=0.973$ ). The content validity was good, with no ceiling or floor effect. The construct, convergent, and divergent validities were good. Moderate responsiveness was evident, with a standardized response mean of 0.74 .

Conclusions: The KKS, has good validity, reliability, and responsiveness. The KKS includes items for floor life style, which are thought more important for Korean patients. The KKS can be used as a good evaluation questionnaire for Korean knee osteoarthritis patients.
\end{abstract}

Key words: Korean Knee score, Knee, Validation, Floor life style.

\section{Introduction}

Many subjective questionnaires for health assessment have been developed to evaluate the patients' status according to the patient's viewpoint, since subjective self-assessment tools are known to be more relevant to the real status of patients than objective measurement methods ${ }^{1)}$.

Received April 10, 2012; Revised May 8, 2012; Accepted July 2, 2012. Correspondence to: Myung Chul Lee, MD, PhD.

Department of Orthopedic Surgery, Seoul National University College of Medicine, 103 Daehak-ro, Jongno-gu, Seoul 110-744, Korea.

Tel: +82-2-2072-3212, Fax: +82-2-764-2718

Email: leemc@snu.ac.kr

This is an Open Access article distributed under the terms of the Creative Commons Attribution Non-Commercial License (http://creativecommons.org/licenses/by-nc/3.0/) which permits unrestricted non-commercial use, distribution, and reproduction in any medium, provided the original work is properly cited.
In assessing health status, different cultures mean different cultural habits, different lingual expression, and different health care systems ${ }^{2}$. Therefore, to perform comprehensive evaluation of health status, questionnaires which reflect the cultural differences should be utilized. Most previous questionnaires have been developed by Western researchers, so Asian researchers have experienced difficulty administering to their patients ${ }^{3-5)}$. Because of that, cross cultural adaptation procedure, the process of translation into another language and modification for cultural configuration, has been attempted by researchers from nonEnglish regions ${ }^{2,6-8)}$. Some investigators have developed novel questionnaire systems suited to their own cultural configuration ${ }^{9)}$. When we focused on knee function, Eastern style of floor life demands considerably different functions compared to the Western style. To sit at the floor or rise from the floor demands a high range of motion, more than 120 degrees ${ }^{10)}$. However, previously developed and used questionnaires, such as Western Ontario and McMaster Universities Arthritis Index (WOMAC), Knee Society Score (KSS) and Knee injury and Osteoarthritis Outcome Score (KOOS), did not give weight to high flexion 
152 Ha et al. Important Symptoms for Korean Patients in Knee Osteoarthritis

Table 1. The Research Committee for Development of a Novel Knee Evaluation System of Korea Knee Society

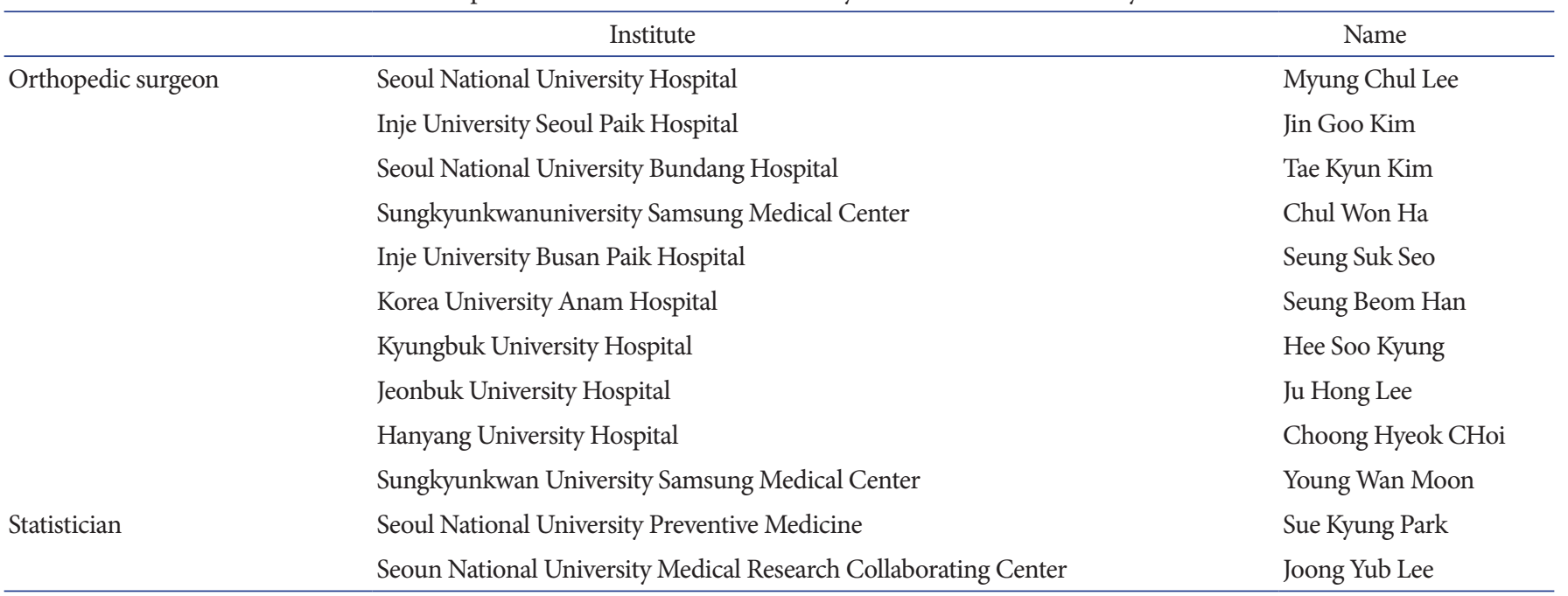

activity and did not include items for high range of motion ${ }^{5,11,12)}$.

Korean researchers have had considerable difficulty using previous scales because they did not reflect the status of Korean people. This raised the need for a novel evaluation system for knee related function, reflective of the floor life style more accurately. Therefore the Korean Knee Score (KKS) was developed by KKS.

The purpose of this study is to report the procedure of developing a new questionnaire, especially focusing on the item generation step, and to validate a novel knee evaluation instrument, the KKS, reflecting a floor life style with high knee flexion. We hypothesized that Korean people regarded knee functions related to floor life style as more important and a newly developed questionnaire would have good measurement properties.

\section{Materials and Methods}

In 2009, the Korean Knee Society launched a special research committee for the development of a novel knee evaluation system, the KKS, for patients with osteoarthritis and a floorbased lifestyle. The committee was composed of 10 orthopedic specialists, who represented active clinical centers for osteoarthritis, and two statisticians (Table 1). The development and validation of the new scale followed the guidelines of the International Society of Arthroscopy, Knee Surgery and Orthopaedic Sports Medicine (ISAKOS), which present two phases and nine steps for the development and testing of diseasespecific quality of life (QOL) measurement ${ }^{13)}$ (Fig. 1).

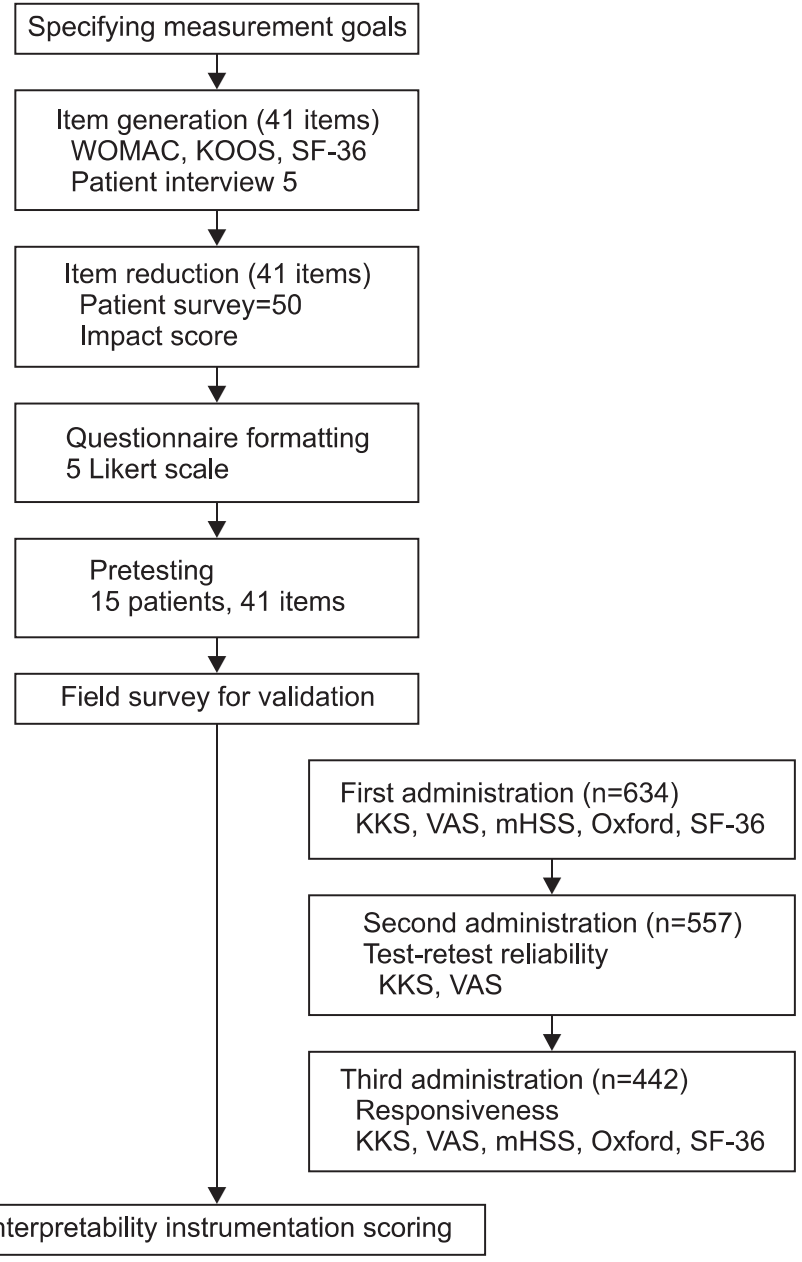

Fig. 1. Flow chart of the developmental procedures of the KKS WOMAC: Western Ontario and McMaster Universities Arthritis Index, KOOS: Knee injury and Osteoarthritis Outcome Score, SF-36: Short form-36, KKS: Korean Knee score, VAS: Visual Analog Scale, mHSS: modified Hospital for Special Surgery score. 
Table 2. Structure of the Korean Knee Score, Forty One Items Are Listed with Their Subdomain and Their Origin

\begin{tabular}{|c|c|c|}
\hline Subdomain & Items & Origin \\
\hline \multirow{12}{*}{$\begin{array}{l}\text { Pain and } \\
\text { symptoms }\end{array}$} & Nocturnal pain & $\mathrm{W}$ \\
\hline & Pain on sitting or lying & $\mathrm{W}$ \\
\hline & Pain on standing & $\mathrm{W}$ \\
\hline & Pain on using stairs & $\mathrm{W}$ \\
\hline & Pain during walking & $\mathrm{W}$ \\
\hline & Pain on straightening fully & $\mathrm{K}$ \\
\hline & Pain on bending fully & K \\
\hline & Morning stiffness & $\mathrm{W}$ \\
\hline & Stiffness at afternoon & $\mathrm{W}$ \\
\hline & Swelling & $\mathrm{K}$ \\
\hline & Feeling grinding or clicking & K \\
\hline & Catching or hang up & $\mathrm{K}$ \\
\hline \multirow{17}{*}{$\begin{array}{l}\text { Physical } \\
\text { functions }\end{array}$} & Descending stairs & $\mathrm{W}$ \\
\hline & Ascending stairs & $\mathrm{W}$ \\
\hline & Rising from sitting & $\mathrm{W}$ \\
\hline & Standing & W \\
\hline & Bending & $\mathrm{W}$ \\
\hline & Walking & $\mathrm{W}$ \\
\hline & Getting in/out of a car & $\mathrm{W}$ \\
\hline & Shopping & $\mathrm{W}$ \\
\hline & Putting on socks & W \\
\hline & Taking off socks & $\mathrm{W}$ \\
\hline & Rising from bed & $\mathrm{W}$ \\
\hline & Lying in bed & W \\
\hline & Getting in/out of bath & $\mathrm{W}$ \\
\hline & Sitting & $\mathrm{W}$ \\
\hline & Getting on/off toilet & $\mathrm{W}$ \\
\hline & Heavy household duties & $\mathrm{W}$ \\
\hline & Light household duties & W \\
\hline \multirow{6}{*}{$\begin{array}{l}\text { Evaluation of } \\
\text { floor life }\end{array}$} & Kneeling pain & $\mathrm{N}$ \\
\hline & Pain on squatting & $\mathrm{N}$ \\
\hline & Pain on standing up from the floor & $\mathrm{N}$ \\
\hline & Pain on sitting with crossed leg on the floor & $\mathrm{N}$ \\
\hline & Difficulty on sitting with crossed legs & $\mathrm{N}$ \\
\hline & Difficulty on rising from sofa or low chair & $\mathrm{N}$ \\
\hline \multirow{6}{*}{$\begin{array}{l}\text { Socio-emotional } \\
\text { function }\end{array}$} & Awareness & K \\
\hline & Life style modification & $\mathrm{K}$ \\
\hline & Lack of confidence & $\mathrm{K}$ \\
\hline & General difficulties & $\mathrm{K}$ \\
\hline & Social functioning & S \\
\hline & Travelling & $\mathrm{N}$ \\
\hline
\end{tabular}

W: Western Ontario and McMaster Universities Arthritis Index, K: Knee injury and Osteoarthritis Outcome Score, S: Short form-36, N: newly developed.

\section{Item Generation}

We followed three steps for item generation. First, the committee members reviewed the literature and selected items from previous instruments: WOMAC, KOOS, and Short form36 (SF-36). Second, the 10 experts on the committee provided their opinions and seven novel items were generated. The most important step is interviewing patients with osteoarthritis. Five such patients were interviewed and asked to identify how their condition interfered with their QOL. This step did not result in the generation of new items.

Finally, 41 items were generated (Table 2) and the items were divided into four subdomains: pain and symptoms, physical function, evaluation of floor life, and socioemotional function. Twelve items were in the pain and symptoms subdomain: seven were selected from the WOMAC and five were from the KOOS. The physical function subdomain contained 17 items, all of which were from the WOMAC. The evaluation of floor-based lifestyle subdomain included six items, all of which were newly generated by the committee members. The socioemotional function subdomain contained six items: four were from the KOOS, one was from the SF-36, and one item was added by the committee.

\section{Item Reduction}

To reduce the number of items, the authors surveyed 50 patients and asked two questions for each item: 'Have you ever experienced this problem?' 'How important is this item to you?' The frequency was determined as the proportion of patients experiencing a particular item and the importance was calculated the mean importance score as rated by the patients. Impact scores were calculated by multiplying the frequency by the mean importance score of each item.

\section{Measurement Properties \\ 1) Reliability}

We evaluated two measures of reliability: test-retest reliability and internal consistency. Test-retest reliability, often called reproducibility or repeatability, measures whether the instrument produces the same results on repeated administration to stable subjects ${ }^{13}$, and was determined using intraclass correlation coefficients (ICCs). Internal consistency was assessed using Cronbach's alpha, which addresses the homogeneity of the questions within a questionnaire.

\section{2) Responsiveness}

Responsiveness, or the ability to reflect changes in patient status, 
was assessed for the KKS using standardized response means (SRMs) calculated by the following formula: (mean postoperative score - mean preoperative score)/standard deviation of the change in score. High responsiveness indicates a greater ability to detect changes ${ }^{14)}$.
3) Validity

The validity of an instrument is defined as "measuring what it is supposed to ${ }^{15}$." Validity refers to the precision with which the 'true value' is estimated by the questionnaire.

(1) Content validity: Content validity refers to the ability of an

Table 3. Impact Scores of Items

\begin{tabular}{|c|c|c|c|}
\hline Rank & Subdomains & Impact score & Items \\
\hline 1 & 2 & 4.18 & Difficulty on heavy household duties \\
\hline 2 & 3 & 4.16 & Pain on sitting with crossed leg on the floor \\
\hline 4 & 3 & 3.28 & Pain on squatting \\
\hline 5 & 2 & 3.2 & Difficulty on getting in/out of a car \\
\hline 7 & 3 & 3.19 & Pain on standing up from the floor \\
\hline 8 & 2 & 3.11 & Difficulty on descending stairs \\
\hline 9 & 2 & 3.11 & Difficulty on ascending stairs \\
\hline 10 & 3 & 3.06 & Difficulty on rising from sofa or low chair \\
\hline 14 & 2 & 2.57 & Difficulty on rising from sitting \\
\hline 15 & 2 & 2.53 & Difficulty on shopping \\
\hline 16 & 1 & 2.53 & Swelling \\
\hline 17 & 2 & 2.41 & Difficulty on bending \\
\hline 18 & 3 & 2.31 & Pain on sitting with crossed leg on the floor \\
\hline 19 & 1 & 2.31 & Morning stiffness \\
\hline 20 & 2 & 2.17 & Difficulties on standing \\
\hline 21 & 2 & 2.06 & Difficulties on walking \\
\hline 22 & 2 & 2.03 & Difficulties on Getting on/off toilet? \\
\hline 28 & 2 & 1.66 & Difficulties on lying in bed \\
\hline 29 & 1 & 1.58 & Pain on straightening fully \\
\hline 30 & 2 & 1.47 & Difficulties putting on socks \\
\hline 31 & 2 & 1.36 & Difficulties on sitting \\
\hline 32 & 2 & 1.34 & Difficulties taking off socks \\
\hline 33 & 1 & 0.97 & Nocturnal pain \\
\hline 34 & 1 & 0.94 & Pain on sitting \\
\hline 35 & 1 & 0.86 & Catching or hang up \\
\hline
\end{tabular}

Subdomain: 1, pain and symptoms; 2, physical functions; 3 , evaluation of floor life style. 
instrument to represent all important areas of the construct of interest. The floor effect is the percentage of subjects with the lowest possible score, and the ceiling effect is the percentage of those with the highest possible score. A floor or ceiling effect less than $20 \%$ indicates good content validity.

(2) Construct validity: Construct validity, comprising convergent and divergent validity, reflects the explanatory power in relation to established measures. Convergent validity refers to the degree to which a measure is correlated with other theoretically correlated measures, and divergent validity refers to the degree to which a measure is not correlated with other measures with predicted non-correlation. The construct validity of the KKS was tested by calculating Pearson's correlation coefficients with the Oxford Knee Score (OKS), modified Hospital for Special Surgery Score (m-HSS), and SF-36 score.

\section{Results}

\section{Impact Score for Reduction of Items}

Fifty patients were included in the survey for impact score calculation. Thirty five (items of socio-emotional subdomain were excluded) items were administered to the patients. The impact score of each item was obtained by the method described above and the items were listed in order of the score (Table 3). Most items from the evaluation of floor life subdomain ranked highly. Five of 6 items from subdomain 3 (evaluation of floor life) ranked within top 10. Mean value of impact score of each subdomain were 1.95 in pain and symptom subdomain, 2.34 in physical function subdomain, and 3.22 in evaluation of floor

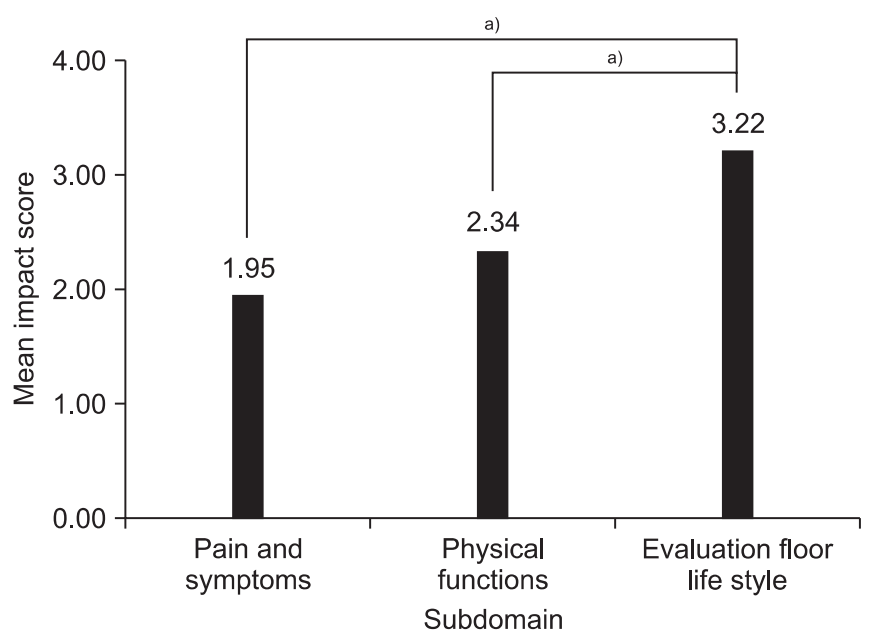

Fig. 2. Comparisons of impact scores among the three subdomains: pain and symptoms, physical functions, evaluation of floor life style. ${ }^{\text {a) }} \mathrm{p}<0.05$ with Scheffe post hoc analysis after analysis of variance. life subdomain. There were significant differences between the evaluation of floor subdomain and the others (analysis of variance with Scheffe post hoc analysis, $\mathrm{p}<0.05$ ) (Fig. 2). It was found that the patients thought knee functions related to floor life style were more important than other functions.

\section{Validation}

1) Demographic data

Nationwide, 634 patients (70 men, 564 women) in 10 institutes participated in this survey (Table 1). The second survey to assess test-retest reliability included 557 participants and the third survey to assess responsiveness included 442 participants.

\section{2) Reliability}

The authors administered the KKS and Visual Analog Scale (VAS) for pain during the first and second surveys. Only those

Table 4. Measurement Properties of the Korean Knee Score

\begin{tabular}{ll}
\hline \multicolumn{1}{c}{ Measurement properties } & Value \\
\hline Validity & \\
Construct validity (correlation coefficient) & \\
Oxford Knee Score & 0.871 \\
Modified Hospital for Special Surgery & \\
$\quad$ Knee score & 0.599 \\
$\quad$ Function score & 0.690 \\
Short form-36 & \\
$\quad$ Total & 0.695 \\
$\quad$ General health & 0.320 \\
$\quad$ Physical functioning & 0.668 \\
$\quad$ Role-physical & 0.572 \\
$\quad$ Bodily pain & 0.719 \\
$\quad$ Vitality & 0.402 \\
Social functioning & 0.549 \\
Role-emotional & 0.407 \\
Mental health & 0.428 \\
Content validity & \\
Ceiling effect/floor effect & \\
Reliability & \\
Internal consistency & \\
\hline Standardized response mean & \\
\hline
\end{tabular}


subjects with the same VAS pain scores in both surveys were included in the reliability analysis (Table 4). The KKS showed excellent test-retest reliability (ICC $=0.931 ; \mathrm{p}<0.01)$ and internal consistency (Cronbach's $\alpha=0.973$ ). Internal consistency could not be increased by the deletion of any item.

\section{3) Responsiveness}

The calculated SRM of the KKS was 0.74, which was higher than that of any other instrument.

\section{4) Validity}

(1) Content validity: The KKS showed good content validity (Table 4). The scores were normally distributed, and no ceiling or floor effect was observed.

(2) Construct validity: The KKS showed good correlation with the m-HSS knee and function scores ( $\mathrm{r}=0.599$ and 0.690 , respectively), the OKS ( $\mathrm{r}=0.871$ ), and the physical functioning, role-physical, bodily pain, and social functioning domains of the SF-36. Weak correlations were obtained with the general health, role-emotional, vitality, and mental health domains of the SF-36. Both convergent and divergent validities were good.

\section{General Description of the Final Form of the KKS}

The KKS consists of 41 items with 4 subdomains. Each item has 0 to 4 point scores, thus, the highest possible score is 164 and lowest possible score is 0 . This raw score should be converted into 0 to 100 scale as follows: $\mathrm{KKS}=($ total score/164) $\times 100$, This score is interpreted as a measure of function such that higher scores represent higher levels of function and lower levels of symptoms.

\section{Discussion}

The principle finding of this study was that the Korean patients thought that knee functions related to floor life style were more important than other functions. The evaluation of floor life style subdomain has the questions, which are kneeling pain, pain on squatting, pain on standing up from the floor, pain on sitting with crossed legs on the floor, difficulty on sitting with crossed legs, and difficulty on rising from a sofa or low chair. Although these questions were generated from the expert committee of the Korea Knee Society, we want to emphasize that the importance of these questions are not just derived from the experts' personal ideas, but also confirmed by the patients during the developing procedure. Impact scores of these items were higher than other items.

The results of the present study demonstrate that the KKS can be used a good evaluation questionnaire for the Korean Knee osteoarthritis patients. The KKS meets the criteria of reliability, validity, responsiveness, and amendability for cross-study comparisons with existing tools. The KKS showed high test-retest reliability, indicating adequate standardization and adaptation to patients. The items comprising the KKS also showed high internal consistency, and each item score showed an excellent correlation with the total KKS score. Content validity was confirmed by the normal distribution of the KKS responses of all 634 patients and the absence of floor and ceiling effects. The KKS demonstrated good correlation with established scoring systems, confirming construct validity. As expected, the highest correlation occurred between SF-36 and KKS scales that measured similar constructs (physical functioning, role-physical, bodily pain, and social functioning). Generally, higher correlations were detected when KKS scales were compared with SF-36 scales that measured physical health (good convergent construct validity), and lower correlations were found between KKS and SF-36 scales that measured mental health (good divergent construct validity). The KKS yielded a moderate SRM value at 3 months ( $\mathrm{SRM}=0.74$ ), indicating that it is moderately responsive. This value was also found to be higher than those of the other instruments.

In Korea, people would sit more often on the floor than on a chair. Although the living style has been changed into westernized fashion, such as bed, sofa, dining table at home, most Korean people still have many chances to sit on the floor with deep bending of knee joints during daily living. Cross legged sitting is possible with the flexion of 135 degree, Sitting on legs (Seiza sitting in Japanese, pray in Muslim way, tea ceremony in Korea, pray in Buddhism way, and so on, commonly seen in Eastern life style) is possible with knee flexion of 150 degree. Standing from the floor is very difficult with the 90 degrees of flexion ${ }^{10)}$. This activity is not only related to the functional aspect, but also to the quality of life. People are normally happier to be able to live in a traditional lifestyle in their society ${ }^{10}$.

Different cultures have different health care systems, different ways for people to solve the health problems, and different disease expressions. Therefore, to use a scale in a different country in a different culture and language, cross cultural adaptation must be undertaken ${ }^{2}$. Among the knee scales, WOMAC, KOOS, KSS, which were developed and validated by Western researchers, did not include items for floor life style. Therefore, these questionnaires cannot reflect the real status of knee function of the people with floor based life style in Eastern countries. Cultural adaptation of them is not sufficient to meet the need to evaluate the knee function with high-flexion. Because of these, 
the need for developing a novel evaluation questionnaire for floor life style has emerged.

The committee for development of a Korean Knee Score was launched in 2009 by the Korea Knee Society. The committee decided to develop a novel knee evaluation system which could be applied to osteoarthritis of the knee, designed as a patientderived assessment system and includes questions regarding Health related QOL (HRQOL). Importantly, this new instrument aims to reflect a floor based lifestyle with high knee flexion.

There are several limitations in our validation procedures. The committee should continue further studies to overcome the limitations and promote wide spread use of the KKS. First, the KKS was validated to osteoarthritis patients, who were treated only by conservative methods. Application of the KKS to a variety of patients who undertake non-operative treatment, arthroscopic surgery, and arthroplasty surgery should be conducted. In addition, there are four subdomains in the scale. We will investigate each subdomain's measurement properties. By comparison with previously developed questionnaire (WOMAC, KOOS, KSS, et al.), characteristics of the KKS can be verified.

\section{Conclusions}

The authors developed the Korean Knee Score, which had good validity, reliability, and responsiveness. The KKS include items for floor life style, which are thought to be more important for the Korean patients than other items. The KKS can be used as a good evaluation questionnaire for the Korean Knee osteoarthritis patients.

\section{References}

1. Garratt AM, Brealey S, Gillespie WJ; DAMASK Trial Team. Patient-assessed health instruments for the knee: a structured review. Rheumatology (Oxford). 2004;43:1414-23.

2. Guillemin F, Bombardier C, Beaton D. Cross-cultural adaptation of health-related quality of life measures: literature review and proposed guidelines. J Clin Epidemiol. 1993;46:1417-32.

3. Irrgang JJ, Anderson AF, Boland AL, Harner CD, Kurosaka M, Neyret P, Richmond JC, Shelborne KD. Development and validation of the international knee documentation committee subjective knee form. Am J Sports Med. 2001;29:600-13.

4. Roos EM, Lohmander LS. The Knee injury and Osteo- arthritis Outcome Score (KOOS): from joint injury to osteoarthritis. Health Qual Life Outcomes. 2003;1:64.

5. Bellamy N, Buchanan WW, Goldsmith CH, Campbell J, Stitt LW. Validation study of WOMAC: a health status instrument for measuring clinically important patient relevant outcomes to antirheumatic drug therapy in patients with osteoarthritis of the hip or knee. J Rheumatol. 1988;15:1833-40.

6. Haverkamp D, Sierevelt IN, Breugem SJ, Lohuis K, Blankevoort L, van Dijk CN. Translation and validation of the Dutch version of the International Knee Documentation Committee Subjective Knee Form. Am J Sports Med. 2006;34:1680-4.

7. Padua R, Bondi R, Ceccarelli E, Bondi L, Romanini E, Zanoli G, Campi S. Italian version of the International Knee Documentation Committee Subjective Knee Form: cross-cultural adaptation and validation. Arthroscopy. 2004;20:819-23.

8. Bizzini M, Gorelick M. Development of a German version of the knee outcome survey for daily activities. Arch Orthop Trauma Surg. 2007;127:781-9.

9. Tae SK, Rhee YG, Park TS, Lee KW, Park JY, Choi CH, Koh SH, Oh JH, Kim SY, Shin SJ. The development and validation of an appraisal method for rotator cuff disorders: the Korean Shoulder Scoring System. J Shoulder Elbow Surg. 2009;18:689-96.

10. Ueo T, Kihara Y, Ikeda N, Kawai J, Nakamura K, Hirokawa S. Deep flexion-oriented bisurface-type knee joint and its tibial rotation that attributes its high performance of flexion. J Arthroplasty. 2011;26:476-82.

11. Roos EM, Roos HP, Lohmander LS, Ekdahl C, Beynnon BD. Knee Injury and Osteoarthritis Outcome Score (KOOS): development of a self-administered outcome measure. J Orthop Sports Phys Ther. 1998;28:88-96.

12. Insall JN, Dorr LD, Scott RD, Scott WN. Rationale of the Knee Society clinical rating system. Clin Orthop Relat Res. 1989;(248):13-4.

13. Kirkley A, Griffin S. Development of disease-specific quality of life measurement tools. Arthroscopy. 2003;19:1121-8.

14. Beaton DE. Understanding the relevance of measured change through studies of responsiveness. Spine. 2000;25: 3192-9.

15. Guyatt G, Mitchell A, Irvine EJ, Singer J, Williams N, Goodacre R, Tompkins C. A new measure of health status for clinical trials in inflammatory bowel disease. Gastroenterology. 1989;96:804-10. 